\title{
Gene regulatory network construction identified NFYA as a diffuse subtype-specific prognostic factor in gastric cancer
}

\author{
BIN CAO $^{1 *}$, YU ZHAO $^{1 *}$, ZHENG ZHANG $^{1}$, HENGCUN LI $^{1}$, JIE XING $^{1}$, SHUILONG GUO $^{1}$, \\ XINTAO QIU ${ }^{2}$, SHUTIAN ZHANG ${ }^{1}$, LI MIN ${ }^{1}$ and SHENGTAO ZHU ${ }^{1}$ \\ ${ }^{1}$ Department of Gastroenterology, Beijing Friendship Hospital, Capital Medical University, \\ National Clinical Research Center for Digestive Disease, Beijing Digestive Disease Center, \\ Beijing Key Laboratory for Precancerous Lesion of Digestive Disease, Beijing 100050, P.R. China; \\ ${ }^{2}$ Department of Biomedical Informatics, Harvard School of Public Health, Boston, MA 02115, USA
}

Received February 27, 2018; Accepted June 7, 2018

DOI: $10.3892 /$ ijo.2018.4519

\begin{abstract}
Lauren classification is a pathology-based gastric cancer (GC) subtyping system, which is widely used in the clinical treatment of patients with GC. However, genomescale molecular characteristics to distinguish between diffuse (DF) and intestinal (IT) GC remain incompletely characterized, particularly at the transcriptional regulatory level. In the present study, gene regulatory networks were constructed using the Passing Attributes between Networks for Data Assimilation (PANDA) algorithm for DF, IT and mixed GC. The results indicated that $>85 \%$ of transcription factor (TF)-target edges were shared among all three GC subtypes. In TF enrichment analysis, 13 TFs, including nuclear transcription factor Y subunit $\alpha$ (NFYA) and forkhead box L1, were activated in DF GC, whereas 8 TFs, including
\end{abstract}

Correspondence to: $\mathrm{Dr} \mathrm{Li}$ Min or Professor Shengtao Zhu, Department of Gastroenterology, Beijing Friendship Hospital, Capital Medical University, National Clinical Research Center for Digestive Disease, Beijing Digestive Disease Center, Beijing Key Laboratory for Precancerous Lesion of Digestive Disease, 95 Yong'an Road, Beijing 100050, P.R. China

E-mail: minli@ccmu.edu.cn

E-mail: shengtaozhu@126.com

${ }^{*}$ Contributed equally

Abbreviations: GC, gastric cancer; PANDA, Passing Attributes between Networks for Data Assimilation; TF, transcription factor; DF, diffuse; MX, mixed; PPI, protein-protein interaction; OS, overall survival; NFYA, nuclear transcription factor Y subunit $\alpha$; RELA, RELA proto-oncogene; FOXL1, forkhead box L1; SRY, sex determining region Y; NKX3, NK3 homeobox; CREB1, cAMP responsive element binding protein 1; TFAP2A, transcription factor AP-2 $\alpha$; EGR 1 , early growth response $1 ; \mathrm{SP} 1, \mathrm{Sp} 1$ transcription factor; HAND1, heart and neural crest derivatives expressed 1; TLX1, T-cell leukemia homeobox 1

Key words: gene regulatory network analysis, prognosis, gastric cancer, Lauren classification
RELA proto-oncogene and T-cell leukemia homeobox 1 (TLX1), were activated in IT GC. Out of these identified TFs, NFYA [Hazard ratio (HR) $(95 \%$ confidence interval, $\mathrm{CI})=0.560(0.349,0.900), \mathrm{P}=0.017]$ and sex determining region $\mathrm{Y}$ [HR $(95 \% \mathrm{CI})=0.603(0.375,0.969), \mathrm{P}=0.037$ ] were identified as independent prognostic factors in DF GC, but not in IT GC, whereas TLX1 [HR $(95 \%$ CI $)=0.547(0.321,0.9325)$, $\mathrm{P}=0.027]$ was identified as an independent prognostic factor in IT GC, but not in DF GC. Verification at the cellular level was also performed; interference of NFYA expression using small interfering RNA in MGC803 cells (DF GC-derived cells) markedly inhibited cell growth and colony formation. Similar effects were also detected in SGC-7901 cells (IT GC-derived cells), but to a lesser extent. In conclusion, identified gene regulatory networks differed between distinct GC subtypes, in which the same TFs had different biological effects. Specifically, NFYA was identified as a DF subtypespecific independent prognostic factor in GC.

\section{Introduction}

Gastric cancer (GC) is ranked fifth in incidence and third in mortality among all types of cancer worldwide (1). Although the incidence of GC in developed areas has declined in recent decades, it ranks third in incidence and mortality in developing countries, including China (2).

Similar to other carcinomas, GC is a complex disease with extremely high heterogeneity (3). First proposed in 1965, the Lauren system has been widely used in GC classification for over half a century, and is useful in evaluating the natural history of GC carcinogenesis (4-6). Based on pathological morphology, the Lauren system divides GC into intestinal (IT), diffuse (DF) and mixed (MX) GC (4). IT GC is characterized by the formation of gland-like structures of various sizes, the majority of which are highly or moderately differentiated. DF GC is characterized by cancer cell clusters scattered in the gastric wall, without the formation of gland-like structures. In cases where the two types of cancer cells are equivalent in numbers, the cancer is characterized as MX GC (7).

Compared with the World Health Organization (WHO) classification of GC (8), the Lauren system is simpler and 
easier to understand, and has a higher reputability among pathologists (9). Therefore, to the best of our knowledge, more molecular biological studies are based on the Lauren classification system compared with other classification systems, such as the WHO system (10-15). Molecular characteristics at the gene expression level in DF and IT GC have been well identified $(11,12,14,16)$; however, the gene regulatory networks that distinguish between DF and IT GC remain incompletely characterized.

Previous systemic-level network analyses have been widely applied to study disease, which have provided significant insights (17-19). By incorporating numerous sources of data to model biological processes, particularly transcription factor (TF)-based gene regulatory networks, integrative analysis has shown promise in elucidating underlying pathophysiological mechanisms, as well as in the development of novel and precise therapies $(19,20)$.

Among these tools, Passing Attributes between Networks for Data Assimilation (PANDA) exhibits higher performance and accuracy. PANDA predicts TF-target regulatory relationships by combining information from gene expression, protein-protein interaction (PPI) and TF-sequence-motif data, in a message-passing approach (21). PANDA has been used in the study of several diseases, including chronic obstructive pulmonary disease (22), ovarian cancer (23) and triple-negative breast cancer (24).

In our previous study, it was demonstrated that patients with DF and IT GC have differing molecular characteristics at the gene expression level, and Frizzled-related protein, epidermal growth factor-containing fibulin like extracellular matrix protein 1 and keratin 23 were identified as subtypespecific prognostic factors from the analyses of differentially expressed genes (16). In the present study, the molecular differences between DF and IT GC were further evaluated at the TF-target regulatory level using the PANDA algorithm. In addition, gene regulatory networks for the different subtypes of GC were constructed, and the prognostic value of TFs specifically activated in DF or IT GC was determined.

\section{Materials and methods}

Data source and preprocessing. The GSE62254 cDNA microarray dataset was downloaded from the Gene Expression Omnibus website (https://www.ncbi.nlm.nih.gov/geo/). Corresponding patient information, including Lauren classification and survival data, was obtained from the supplementary materials of the original article (25). The Robust Multichip Average algorithm (26) was applied for background correction, and qspline was applied for normalization (27). Data were perfect match-corrected and summarized using the Li-Wong method (28). All probes were mapped to Ensembl Gene Symbols in the R package 'mygene' (29).

TF-target network construction. Position weight matrix data of TF-binding motifs in vertebrates were obtained from the JASPAR database (30). The methods and parameters of binding site scanning used were previously described (31). PPI data were obtained from a publicly available dataset (https://www.ncbi.nlm.nih.gov/pmc/articles/PMC2836267/ bin/NIHMS177825-supplement-03.xls) (32).
Networks were constructed using PANDA software (http:// sourceforge.net/projects/panda-net/) (21). Networks of DF, MX and IT GC subtypes were constructed by combining the corresponding gene expression, TF motif and PPI data with an update parameter of $\alpha=0.25$. Confident TF-target edges were identified by a False Discovery Rate (FDR) of $<0.05$.

Enrichment of subtype-specific TFs and co-target analysis. AnaPANDA software (23) was used to further identify TFs specifically activated in a certain subtype of GC, and the probability cutoff was set to 0.8 to build sub-networks. The hypergeometric distribution model was utilized to evaluate the overlap between genes co-targeted by each two given TFs.

Survival analysis. Overall survival (OS) was the primary endpoint in the present analysis, which was defined as the time from tumor resection to death or last follow-up. The median mRNA expression level of a given gene was chosen as the cutoff to divide patients into two subgroups. Log-rank tests and Kaplan-Meier plots were used to evaluate the difference in OS between subgroups. Cox proportional hazard model was applied for multiple-variants analysis, in which 'backward LR' stepwise logistic regression was used for variable selection.

Cell culturing and siRNA transfection. Human GC cell lines [MGC803 (the cell line used has been authenticated by STR profiling) and SGC-7901] were purchased from the Cancer Institute and Hospital, Chinese Academy of Medical Sciences (Beijing, China). All cells were maintained in Dulbecco's modified Eagle's medium (cat. no. 10-013-CVR; Corning, Inc., Corning, NY, USA) supplemented with $10 \%$ fetal bovine serum (cat. no. 10270-106; Gibco; Thermo Fisher Scientific, Inc.) at $37^{\circ} \mathrm{C}$ in an incubator containing $5 \% \mathrm{CO}_{2}$. Approximately $5 \times 10^{5}$ cells/well were cultured in a 6 -well plate. After $24 \mathrm{~h}$, cells were transfected with small interfering RNAs (siRNAs) (sequences: si-NC, 5'-UUCUCCGAACGUGUCACGUTT-3'; si-NFYA, 5'-CAAACAAUACCACCGUAUUTT-3') using RNA-mate (BioChain Institute, Inc., Newark, CA, USA) (5 $\mu \mathrm{g}$ siRNA $+10 \mu \mathrm{l}$ RNA-mate) for $6 \mathrm{~h}$ at $37^{\circ} \mathrm{C}$ according to the manufacturer's protocol. siRNAs were purchased from Shanghai GenePharma Co., Ltd. (Shanghai, China).

Western blot analysis. Nuclear transcription factor Y subunit $\alpha$ (NFYA) antibody (cat. no. 12981-1-ap, 1:1,000) was purchased from Wuhan Sanying Biotechnology (Wuhan, China). GAPDH antibody (cat. no. A01020, 1:1,000) was purchased from ARP American Research Products, Inc. (Waltham, MA, USA). Proteins were extracted from the cells using lysis buffer (50 mM Tris-HCl, pH 7.4; 10 mM EDTA; 0.5\% NP-40; $1 \%$ Triton $\mathrm{X}-100$ ) and quantified using the bicinchoninic acid method, after which they were separated $(50 \mu \mathrm{g})$ by $10 \%$ SDS-PAGE and transferred to PVDF membranes. The membranes were blocked with $5 \%$ bovine serum albumin (cat. no. A1933; Sigma-Aldrich; Merck KGaA, Darmstadt, Germany) for $1 \mathrm{~h}$ at $37^{\circ} \mathrm{C}$, and were then incubated with primary antibodies for $2 \mathrm{~h}$ at $37^{\circ} \mathrm{C}$, washed with PBS-1\% Tween (PBST) five times ( $5 \mathrm{~min} / \mathrm{wash}$ ), and then incubated with a secondary antibody (cat. no. 7074, 1:2,000; Cell Signaling Technology, Inc.) for $30 \mathrm{~min}$ at $37^{\circ} \mathrm{C}$. Subsequently, the membranes were washed a further three times with PBST ( $5 \mathrm{~min} /$ wash) and 
Table I. Characteristics of patients included in the present study.

\begin{tabular}{|c|c|c|c|c|}
\hline Variable & $\begin{array}{c}\text { Diffuse } \\
n=134\end{array}$ & $\begin{array}{c}\text { Intestinal } \\
\mathrm{n}=146\end{array}$ & $\begin{array}{c}\text { Mixed } \\
n=20\end{array}$ & P-value \\
\hline Age at diagnosis (years) & & & & $<0.001$ \\
\hline Means \pm SD & $58.44 \pm 12.53$ & $64.41 \pm 9.61$ & $67.35 \pm 7.90$ & \\
\hline Sex & & & & 0.001 \\
\hline Male & 74 & 110 & 15 & \\
\hline Female & 60 & 36 & 5 & \\
\hline Tumor location & & & & 0.382 \\
\hline Cardia & 17 & 12 & 3 & \\
\hline Body & 54 & 54 & 5 & \\
\hline Antrum & 63 & 80 & 12 & \\
\hline MLH1 expression ${ }^{a}$ & & & & 0.083 \\
\hline Positive & 112 & 109 & 13 & \\
\hline Negative & 22 & 35 & 7 & \\
\hline Recurrence & & & & 0.079 \\
\hline Yes & 66 & 49 & 10 & \\
\hline No & 62 & 86 & 9 & \\
\hline Unknown & 6 & 11 & 1 & \\
\hline Stage $^{a}$ & & & & $<0.001$ \\
\hline $\mathrm{I} / \mathrm{II}$ & 39 & 79 & 8 & \\
\hline III & 49 & 37 & 9 & \\
\hline IV & 46 & 28 & 3 & \\
\hline
\end{tabular}

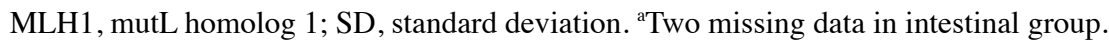

proteins were detected using an enhanced chemiluminescence kit (cat. no. 34076; Thermo Fisher Scientific, Inc.).

Cell proliferation analysis and colony formation assay. For the cell proliferation analysis, a total of $12 \mathrm{~h}$ post-transfection, MGC803 and SGC-7901 cells were cultured in 96-well plates at a density of $2 \times 10^{3}$ cells/well. Cell viability was measured using a Cell Counting kit-8 (CCK-8; Dojindo Molecular Technologies, Inc., Kumamoto,Japan), according to the manufacturer's protocol. For colony formation assay, MGC803 and SGC-7901 cells were cultured in 6 -well plates at a density of $1 \times 10^{3}$ cells/well. After 14 days, colonies were fixed in methanol, stained with $0.25 \%$ crystal violet for $10 \mathrm{~min}$ at room temperature and counted. All assays were repeated three times independently.

Statistical analysis and R package usage. Categorical, baseline data were compared with Pearson's $\chi^{2}$ test or Fisher's exact test, and continuous baseline data were compared with one-way analysis of variance followed by Student Nerman Keuls test in Table I. For comparisons of continuous data, an unpaired Student's t-test was conducted. Wald test was performed to evaluate the overall multivariate Cox model. For all statistical analyses, $\mathrm{P}<0.05$ was considered to indicate a statistically significant difference, and a cutoff value of FDR $<0.05$ was used for multiple comparison corrections. All statistical analyses were two-sided and performed using R Software 3.3.1 (www.r-project.org). R packages 'VennDiagram' and 'ggplot2' were used for data visualization; Mygene was used for gene symbol mapping; MASS and survival were used for survival analysis; q value was used for FDR analysis.

\section{Results}

Baseline characteristics in the three groups. A total of 300 patients were included in the present analysis. Patient characteristics are presented in Table I. In total, there were 134 patients in the DF group, 146 in the IT group and 20 in the MX group. The age in each group was 58.44 $\pm 12.53,64.41 \pm 9.61$ and $67.35 \pm 7.90$ years, respectively. A larger proportion of patients were male (66.33\%). Patients in the DF, IT and MX groups were similar with regards to tumor location, mutL homolog 1 expression and recurrence.

Building TF-target regulatory networks of DF and IT GC subtypes. Expression data were extracted from $134 \mathrm{DF}$, $20 \mathrm{MX}$ and 146 IT GC samples from the GSE62254 dataset. Combining TF motif and PPI data, TF-target regulatory networks for these three subtypes of GC were generated using PANDA software (Fig. 1). For each TF-target edge in each subtype, a Z-score was calculated based on the confidence level of the potential regulatory relationship. Edge Z-score distributions of the various subgroups of GC are presented in Fig. 2A. Different subgroups were assigned different colors.

Identification of DF-and IT-specific TF-target regulatory edges. All edges with an FDR-adjusted P-value of $<0.05$ 


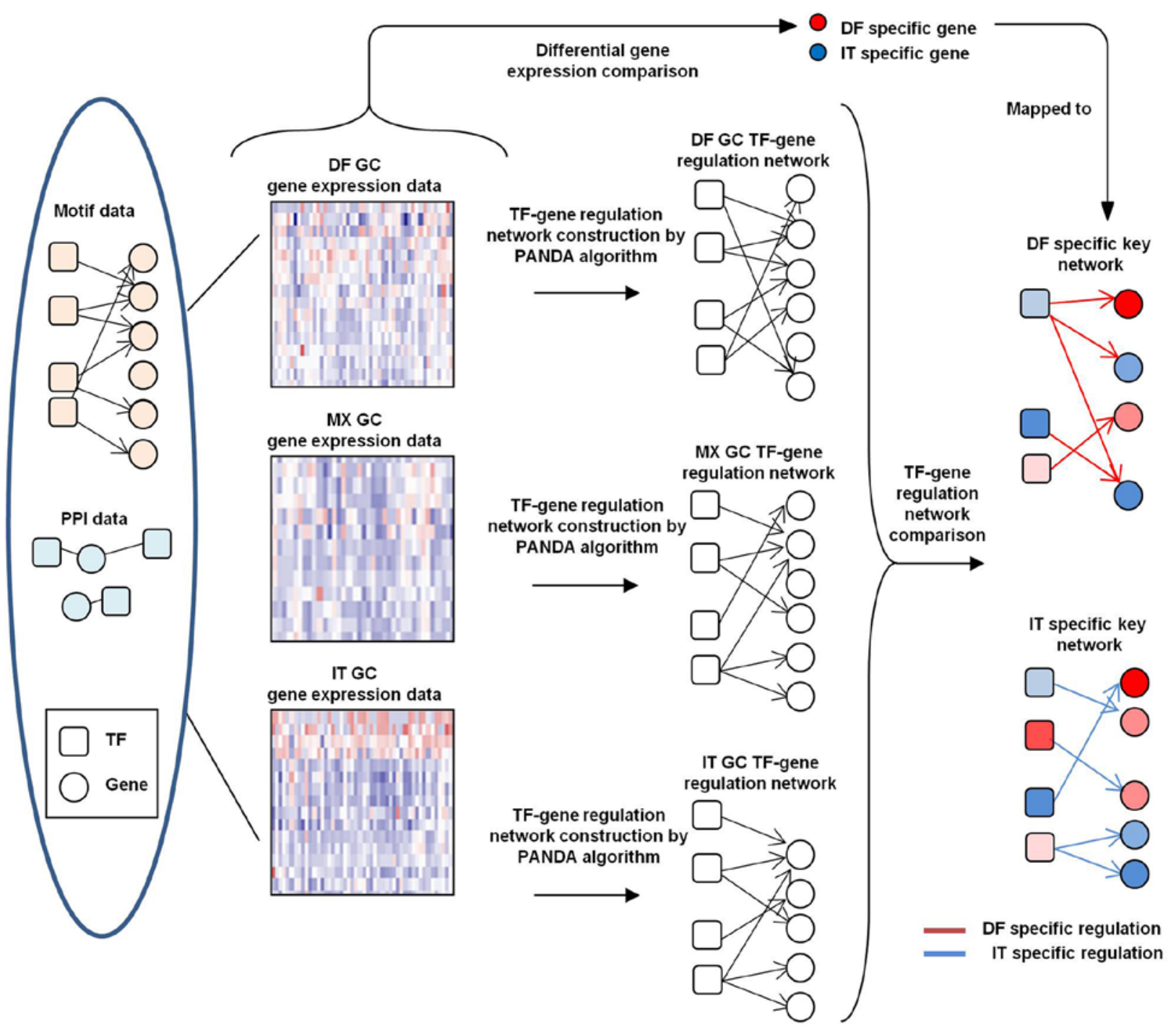

Figure 1. Outline of DF- and IT-specific pattern identification. Motif and PPI data were integrated with gene expression data using the PANDA algorithm, to generate TF-target regulation networks for the different subtypes of GC. Differentially expressed genes were subsequently mapped to these networks, and DF-and IT-specific patterns were identified. DF, diffuse; GC, gastric cancer; IT, intestinal; PANDA, Passing Attributes between Networks for Data Assimilation; PPI, protein-protein interaction; TF, transcription factor.

were considered confident. The overlap of confident edges among the three subtypes of GC was displayed as a Venn diagram (Fig. 2B); $>85 \%$ of TF-target edges were shared among all three subtypes, indicating that the TF-target relationship was strongly conserved. According to the definition of DF-specific edges (ModuleDF), IT-specific edges (ModuleIT) and commonly conserved edges, different edges were marked with different colors in a 3D scatter plot, in which each axis represented a Z-score of each subtype of GC (Fig. 2C). Fig. 2D exhibited the projection of this 3D plot through each axis. The overlap of differentially expressed genes between DF and IT GC, as well as the target genes of ModuleDF and ModuleIT were illustrated in a Venn diagram (Fig. 2E). By applying the hypergeometric distribution model to the target genes of each TF, it was revealed that most TFs with a high activity in ModuleDF also had a high activity in ModuleIT (Fig. 2F).

Enrichment of DF-and IT-specific TFs and co-target analysis. Using the MX subtype as a control, the AnaPANDA algorithm was applied to further identify TFs, which were specifically activated in each subtype of GC. A total of 13 TFs were activated in DF GC (Fig. 3A), and 8 TFs were activated in IT GC (Fig. 3B). Additionally, Fisher's exact test was applied to evaluate the overlap between target genes shared by different pairs of TFs. In DF GC, RELA proto-oncogene (RELA) and forkhead box L1 (FOXL1), sex determining region Y (SRY) and NK3 homeobox (NKX3)-2, NFYA, paired box 2 and cAMP responsive element binding protein 1 (CREB1) were identified as co-targeted, which suggested that those TFs had very similar target profiles (Fig. 3C). In IT GC, NK2 homeobox 5 and NKX3-1, transcription factor AP-2 $\alpha$ (TFAP2A), early growth response 1 (EGR1) and Sp1 transcription factor (SP1) were also identified as co-targeted (Fig. 3D).

Application of enriched TFs as DF- or IT-specific prognostic biomarkers. Among the TFs specifically activated in DF or IT subtypes, TFAP2A, FOXL1 and SP1 were identified as potential prognostic biomarkers in all GC (Fig. 4A-C). NFYA and SRY were identified to be potential prognostic factors in DF GC (Fig. 5A-D), whereas T-cell leukemia homeobox 1 (TLX1) 
A

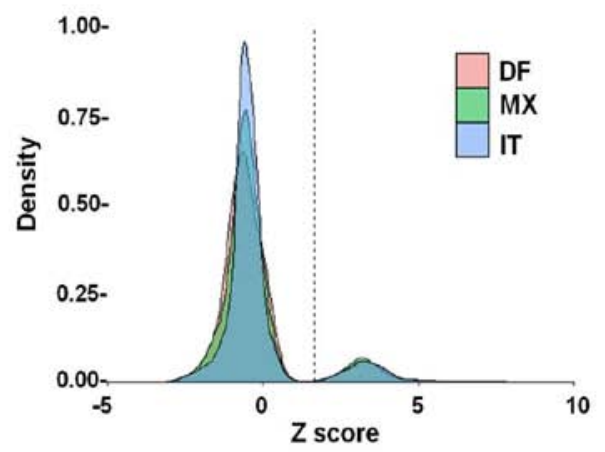

C

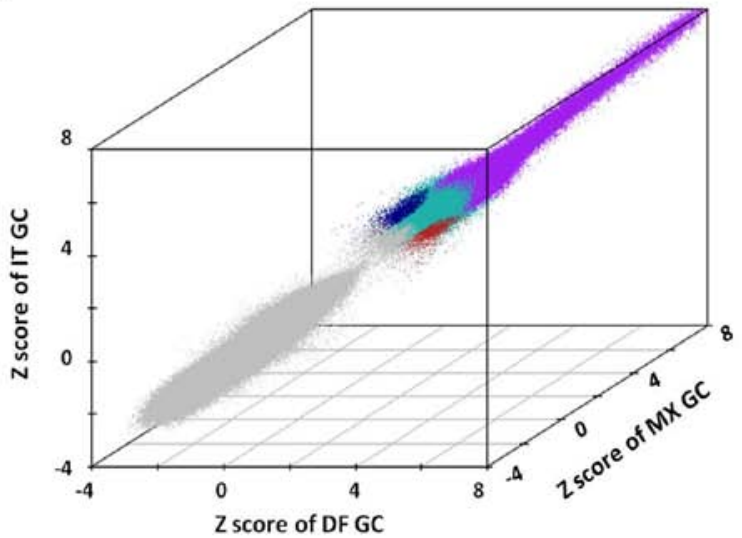

$\mathbf{E}$

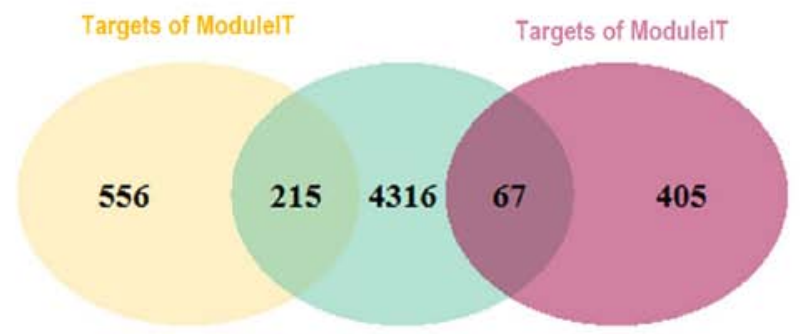

DF IT differentially expressed genes
B
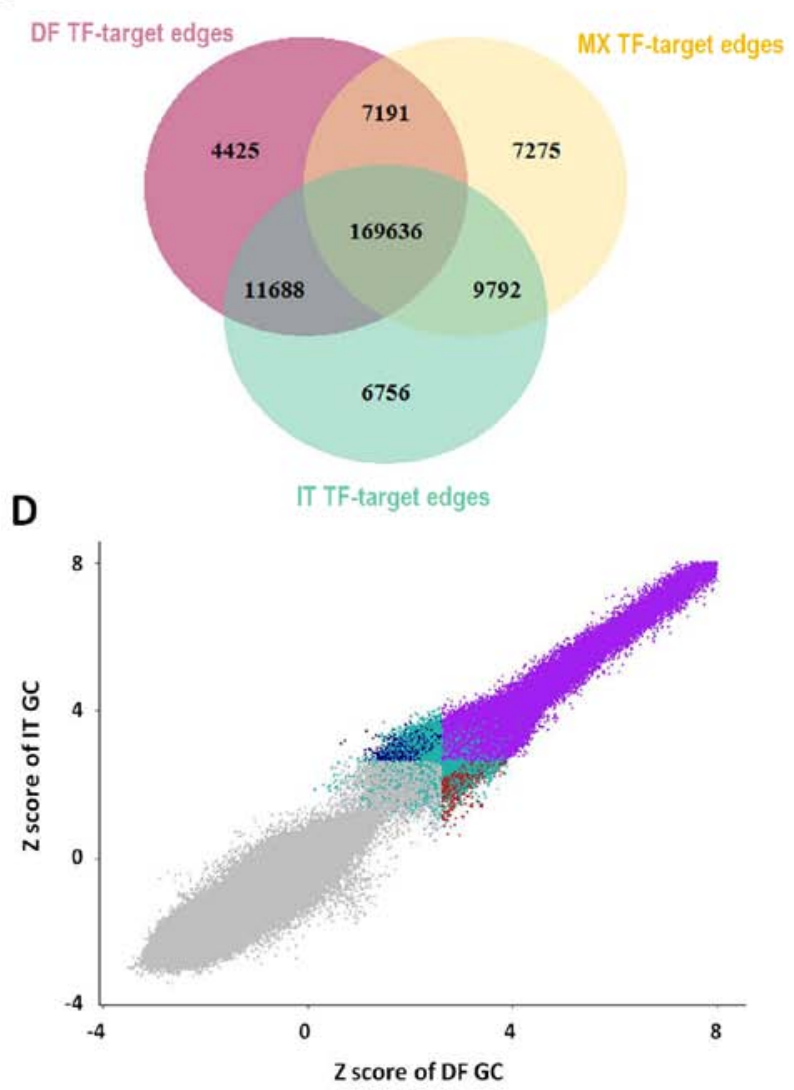

$\mathbf{F}$

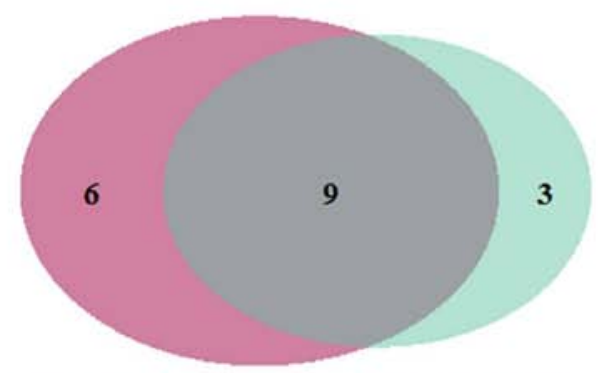

Enriched TF in ModulelT Enriched TF in ModulelT

Figure 2. Gene regulatory network construction, and DF- and IT-specific TF-target edge identification. (A) Edge Z-score distribution in different subgroups of GC. (B) Overlap of TF-target edges among the different subgroups of GC. (C) Identification of DF- and IT-specific TF-target edges in a 3D scatter plot, exhibiting Z scores of DF, MX and IT GC. Low confidence edges were colored gray, conserved edges were colored purple, DF-specific edges were colored red, IT-specific edges were colored blue and other edges were colored green. (D) Projection of 3D view through the y-axis. (E) Overlap of target genes in ModuleDF/ModuleIT and DF/IT differentially expressed genes. (F) Overlap of TFs enriched in DF-specific TF-target edges with DF differentially expressed genes (ModuleDF) and IT-specific TF-target edges with IT differentially expressed genes (ModuleIT). DF, diffuse; GC, gastric cancer; IT, intestinal; MX, mixed; TF, transcription factor.

identified to have potential prognostic value in IT GC (Fig. 5E and F). Meanwhile, heart and neural crest derivatives expressed 1 (HAND1) and CREB1 were also identified to be potential prognostic factors in DF GC (Fig. 6A-D), whereas EGR1 was identified to have potential prognostic value in IT GC (Fig. 6E and F).

Cox proportional hazards model was also applied and respectively implemented for the aforementioned genes. NFYA [hazard ratio (HR) $(95 \%$ confidence interval, $C I)=0.560$ $(0.349,0.900), \mathrm{P}=0.017]$ and SRY $[\mathrm{HR}(95 \% \mathrm{CI})=0.603$ $(0.375,0.969), \mathrm{P}=0.037]$ were identified as independent prognostic factors in DF GC (Tables II and III), whereas TLX1
[HR $(95 \% \mathrm{CI})=0.547(0.321,0.9325), \mathrm{P}=0.027]$ was identified as an independent prognostic factor in IT GC (Table IV). Conversely, EGR1 was not associated with prognosis in either DF or IT GC (Table V).

Investigation of the role of NFYA in DF- and IT-derived GC cells. To confirm the biological function of NFYA in DF and IT GC, NFYA expression was knocked down by siRNA in DF GC-derived MGC803 cells and IT GC-derived SGC-7901 cells (Fig. 7A). CCK-8 assays indicated that knockdown of NFYA expression markedly inhibited the rate of cell growth in DF GC-derived MGC803 cells, and partially inhibited the 
A

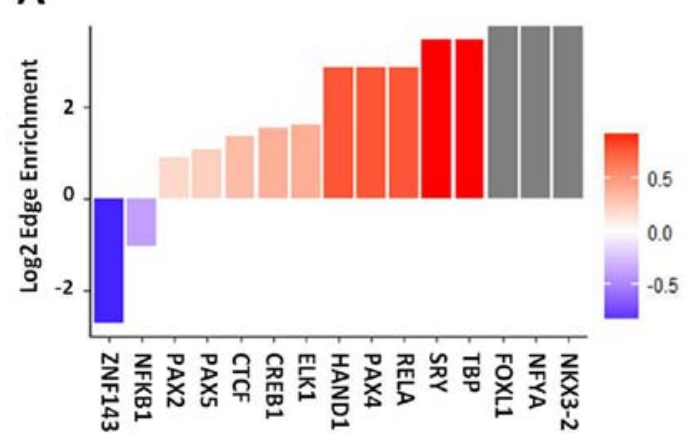

C

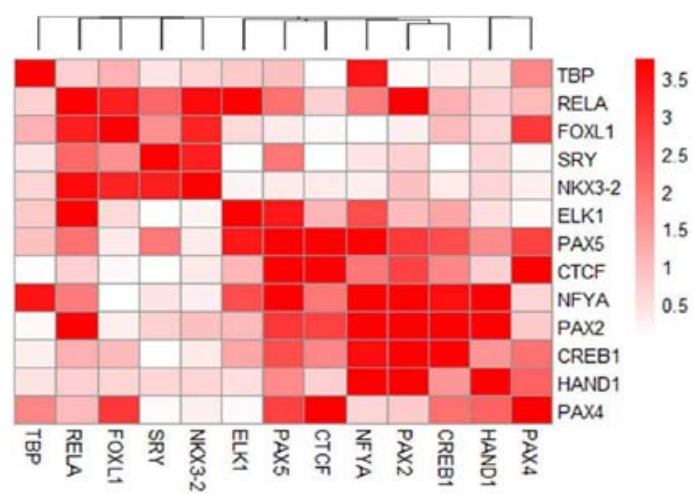

B

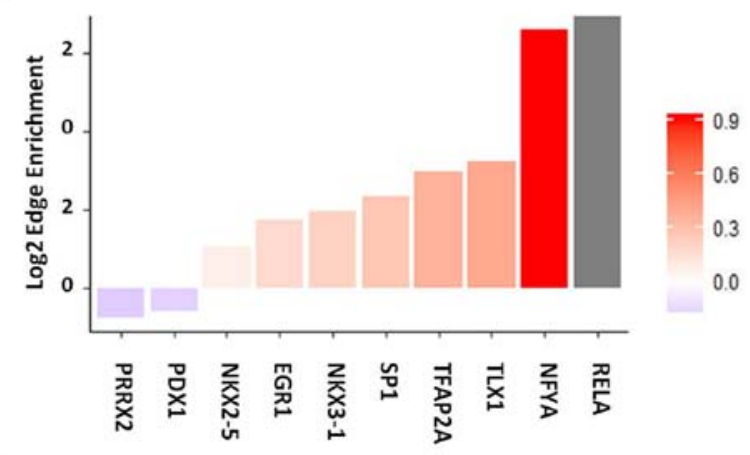

D

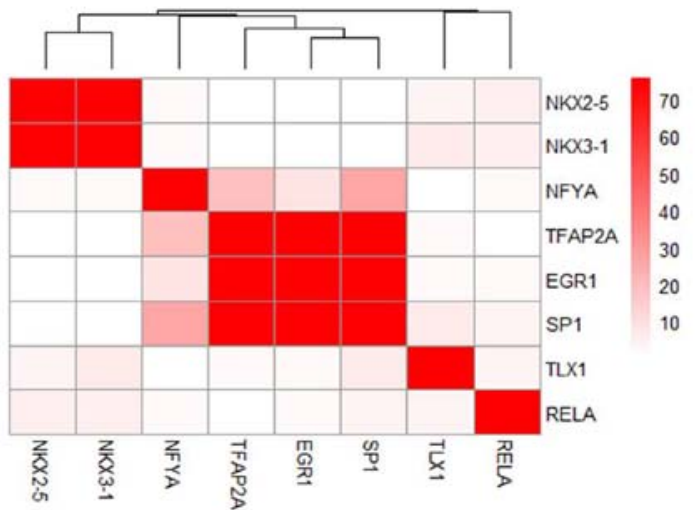

Figure 3. Identification of DF- and IT-specific TFs and co-target analyses. (A) Identification of DF-specific TFs and (B) IT-specific TFs using the AnaPANDA algorithm. (C) Target profile similarity between the DF-specific TFs and (D) IT-specific TFs. DF, diffuse; GC, gastric cancer; IT, intestinal; TF, transcription factor.

A

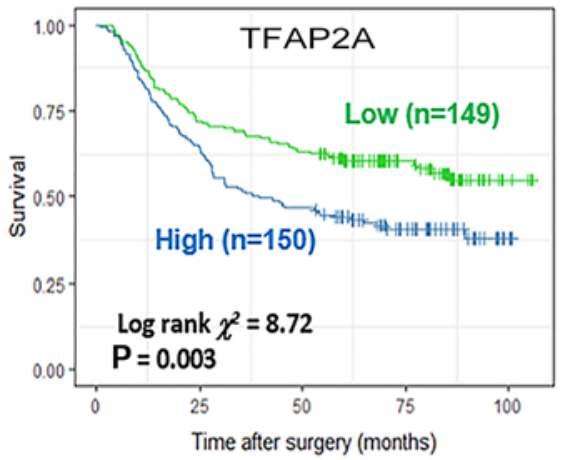

B

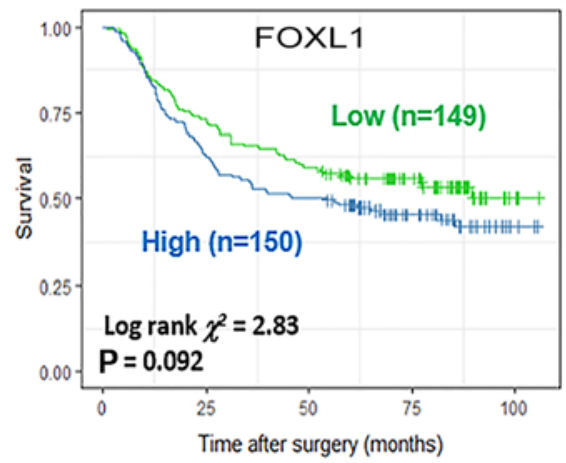

C

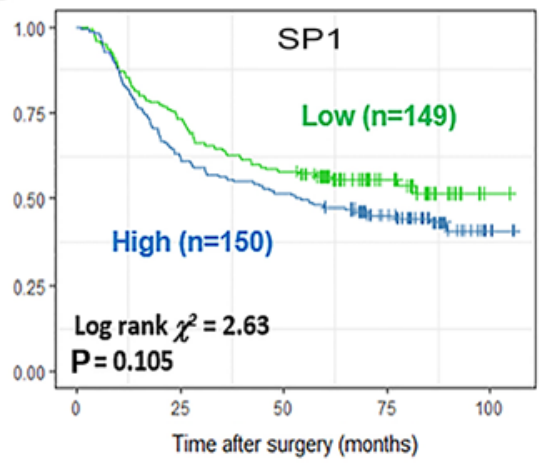

Figure 4. Kaplan-Meier curve for general prognostic biomarker analysis. Kaplan-Meier curves for (A) TFAP2A, (B) FOXL1 and (C) SP1 in gastric cancer. The cutoff was based on the median value of each subgroup. FOXL1, forkhead box L1; SP1, Sp1 transcription factor; TFAP2A, transcription factor AP-2 $\alpha$.

rate of cell growth in IT GC-derived SGC-7901 cells (Fig. 7B). Colony formation assays also demonstrated that the colony formation abilities of DF GC-derived MGC803 cells were nearly eliminated by NFYA siRNA (Fig. 7C). However, the colony formation ability of IT GC-derived SGC-7901 cells was only partially inhibited under the same conditions (Fig. 7C).

\section{Discussion}

The importance of the network construction approach in the study of disease has been highlighted in various reports $(17,18)$. Gene networks have been demonstrated to distinguish disease subtypes with more precision and accuracy, compared with single gene biomarkers (19). Integrative methods incorporating several sources of data to model biological processes, particularly TF-target regulatory networks, have shown promise in providing novel perspectives to understand the underlying pathophysiological mechanisms in disease, as well as in the development of novel and precise therapies $(19,20)$. Numerous tools, including PANDA (21), SEmi-supervised REgulatory Network Discoverer (33), ReMoDiscovery (34), context likelihood of relatedness (35), and C3Net (36), have been developed to integrate data from different levels and construct TF-target regulatory networks. Among all well-known methods, PANDA has been demonstrated to have higher accuracy and performance in a previous study (21).

Although the gene expression patterns of DF and IT GC were investigated in our previous research (16), studies 
Table II. Independent prognostic value of NFYA in diffuse and intestinal gastric cancer.

Diffuse gastric cancer

Variable

NFYA (negative vs. positive)

Sex (male vs. female)

Age

Stage

Stage III vs. I/II

Stage IV vs. I/II

T (3 and 4 vs. 1 and 2)

$\mathrm{N}$ (positive vs. negative)

$\mathrm{M}$ (positive vs. negative)

Overall Cox model

\section{Diffuse gastric cancer}

HR $(95 \% \mathrm{CI}) \quad$ P-value

$0.560(0.349,0.900)$

Variable eliminated

$1.029(1.009,1.049)$

$2.286(1.086,4.812)$

$13.002(6.213,27.210)$

Variable eliminated

Variable eliminated

Variable eliminated

0.017

0.005
Intestinal gastric cancer

HR $(95 \%$ CI)

P-value

Variable eliminated

$2.088(1.040,4.191)$

0.038

$1.059(1.023,1.096)$

0.001

$0.029 \quad$ Variable eliminated

$<0.001 \quad$ Variable eliminated

$3.365(1.956,5.788)$

$<0.001$

$1.483(1.093,2.012)$

0.011

$2.520(0.851,7.460)$

0.095

$<0.001$

$<0.001$

Variable eliminated, variables that were not statistically significant were eliminated in the model. Overall Cox model, the model including all variables that were not eliminated. CI, confidence interval; HR, hazard rate; NFYA, nuclear transcription factor Y subunit $\alpha$.
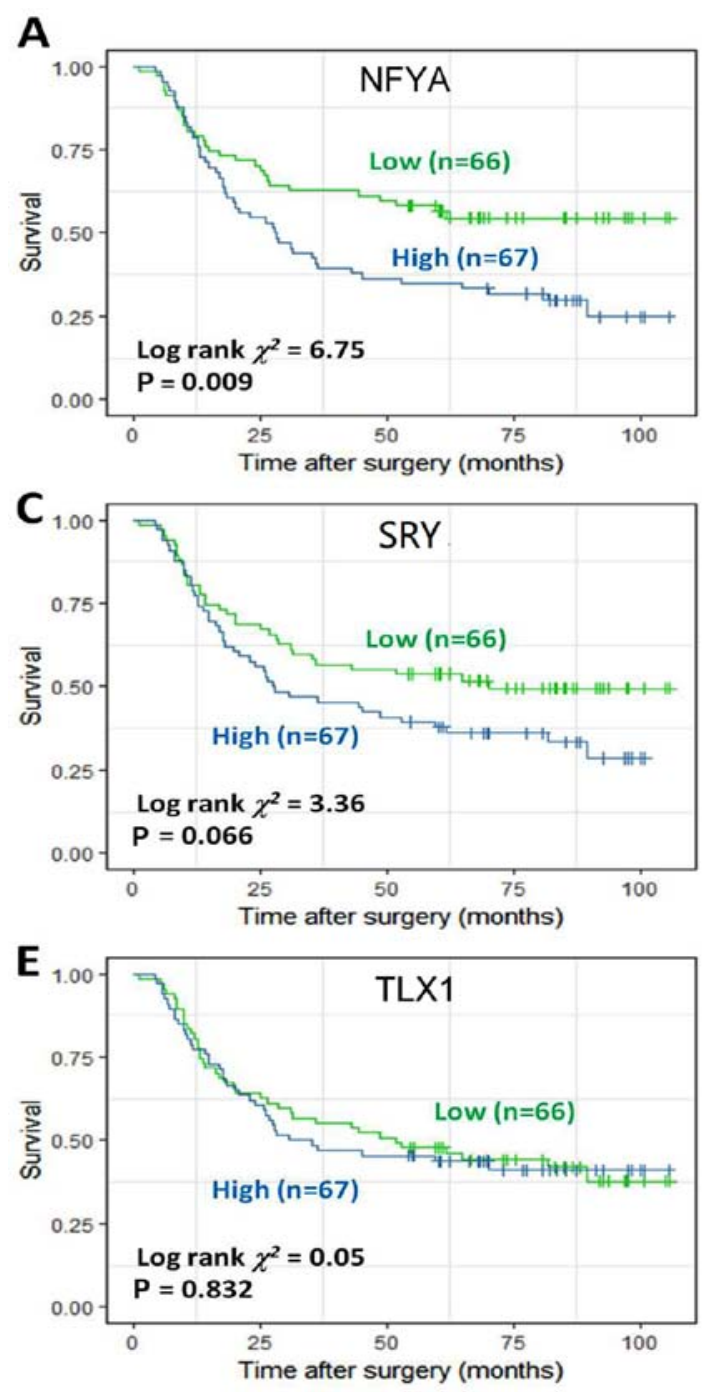

B
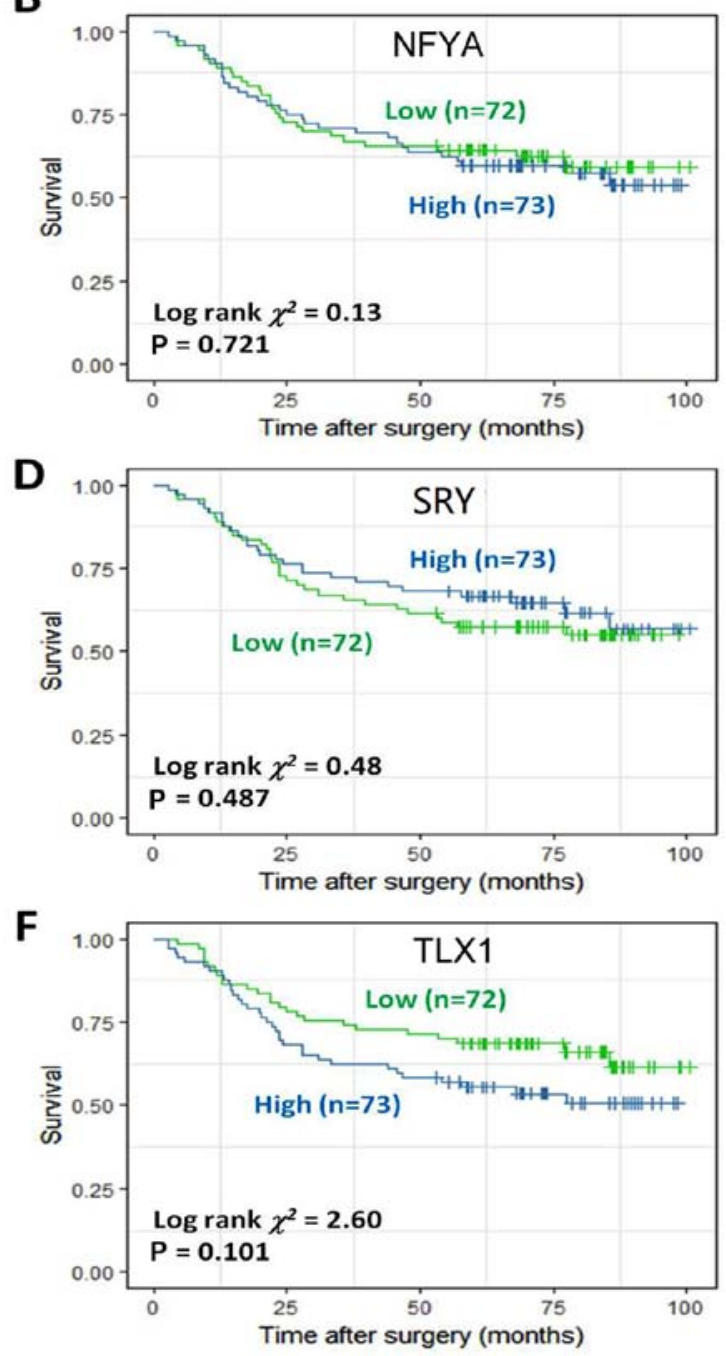

Figure 5. Kaplan-Meier curves for NFYA in (A) diffuse and (B) intestinal GC; Kaplan-Meier curves for SRY in (C) diffuse and (D) intestinal GC; Kaplan-Meier curves for TLX1 in (E) diffuse and (F) intestinal GC. The cutoff was based on the median value of each subgroup. GC, gastric cancer; NFYA, nuclear transcription factor Y subunit $\alpha$; SRY, sex determining region Y; TLX1, T-cell leukemia homeobox 1. 
Table III. Independent prognostic value of SRY in diffuse and intestinal gastric cancer.

Diffuse gastric cancer

Variable

SRY (negative vs. positive)

Sex (male vs. female)

Age

Stage

Stage III vs. I/II

Stage IV vs. I/II

$\mathrm{T}$ (3 and 4 vs. 1 and 2)

$\mathrm{N}$ (positive vs. negative)

$\mathrm{M}$ (positive vs. negative)

Overall Cox model

Variable eliminated

$1.021(1.002,1.040)$

$7.739(2.923,20.494)$

Variable eliminated

$1.421(0.961,2.100)$

Variable eliminated
Intestinal gastric cancer

HR $(95 \%$ CI)

P-value

HR $(95 \% \mathrm{CI}) \quad$ P-value

0.037

0.029

0.129

$<0.001$

0.078

$<0.001$
Variable eliminated

$2.088(1.040,4.191)$

0.038

$1.059(1.023,1.096)$

0.001

Variable eliminated

Variable eliminated

3.365 (1.956, 5.788)

$<0.001$

$1.483(1.093,2.012)$

0.011

$2.520(0.851,7.460)$

0.095

Variable eliminated, variables that were not statistically significant were eliminated in the model. Overall Cox model, the model including all variables that were not eliminated. CI, confidence interval; HR, hazard rate; SRY, sex determining region Y.
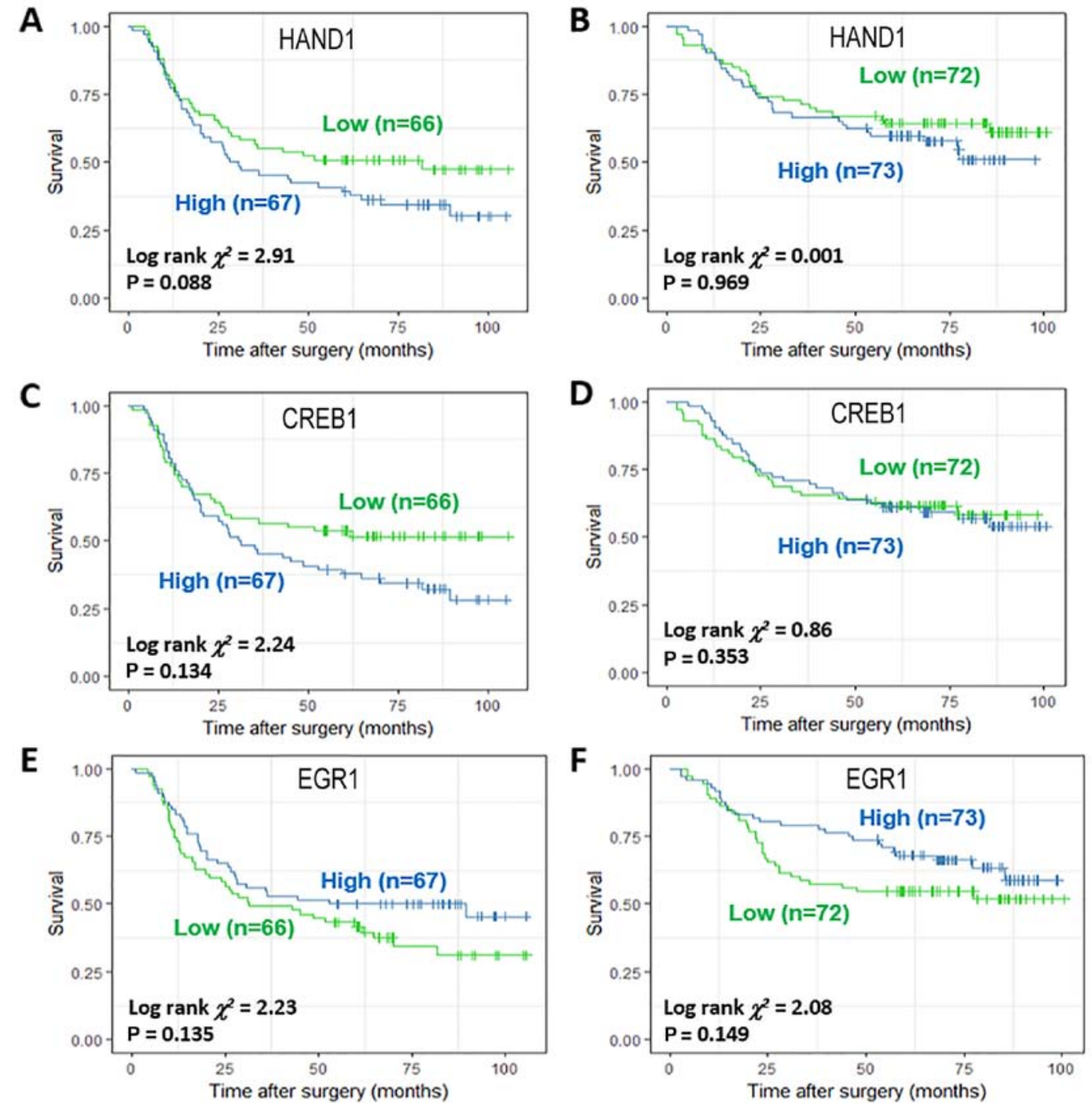

Figure 6. Kaplan-Meier curves for HAND1 in (A) diffuse GC and (B) intestinal GC; Kaplan-Meier curves for CREB1 in (C) diffuse GC and (D) intestinal GC; Kaplan-Meier curves for EGR1 in (E) diffuse GC and (F) intestinal GC. The cutoff was based on the median value of each subgroup. CREB1, cAMP responsive element binding protein 1; EGR1, early growth response 1; GC, gastric cancer; HAND1, heart and neural crest derivatives expressed 1. 
Table IV. Independent prognostic value of TLX1 in diffuse and intestinal gastric cancer.

\begin{tabular}{|c|c|c|c|c|}
\hline \multirow[b]{2}{*}{ Variable } & \multicolumn{2}{|c|}{ Diffuse gastric cancer } & \multicolumn{2}{|c|}{ Intestinal gastric cancer } \\
\hline & HR $(95 \% \mathrm{CI})$ & P-value & $\operatorname{HR}(95 \% \mathrm{CI})$ & P-value \\
\hline TLX1 (negative vs. positive) & Variable eliminated & & $0.547(0.321,0.9325)$ & 0.027 \\
\hline Sex (male vs. female) & Variable eliminated & & $1.924(0.964,3.840)$ & 0.064 \\
\hline Age & $1.023(1.003,1.042)$ & 0.020 & $1.069(1.032,1.107)$ & $<0.001$ \\
\hline \multicolumn{5}{|l|}{ Stage } \\
\hline Stage III vs. I/II & $2.190(1.041,4.610)$ & 0.039 & Variable eliminated & \\
\hline Stage IV vs. I/II & $12.976(6.214,27.099)$ & $<0.001$ & Variable eliminated & \\
\hline $\mathrm{T}$ ( 3 and 4 vs. 1 and 2$)$ & Variable eliminated & & $3.194(1.871,5.453)$ & $<0.001$ \\
\hline $\mathrm{N}$ (positive vs. negative) & Variable eliminated & & $1.645(1.213,2.231)$ & 0.001 \\
\hline M (positive vs. negative) & Variable eliminated & & Variable eliminated & \\
\hline Overall Cox model & & $<0.001$ & & \\
\hline
\end{tabular}

Variable eliminated, variables that were not statistically significant were eliminated in the model. Overall Cox model, the model including all variables that were not eliminated. CI, confidence interval; HR, hazard rate; TLX1, T-cell leukemia homeobox 1.

Table V. Independent prognostic value of EGR1 in diffuse and intestinal gastric cancer.

\begin{tabular}{|c|c|c|c|c|}
\hline \multirow[b]{2}{*}{ Variable } & \multicolumn{2}{|c|}{ Diffuse gastric cancer } & \multicolumn{2}{|c|}{ Intestinal gastric cancer } \\
\hline & $\operatorname{HR}(95 \% \mathrm{CI})$ & P-value & $\mathrm{HR}(95 \% \mathrm{CI})$ & P-value \\
\hline EGR1 (negative vs. positive) & Variable eliminated & & Variable eliminated & \\
\hline Sex (male vs. female) & Variable eliminated & & $2.088(1.040,4.191)$ & 0.038 \\
\hline Age & $1.023(1.003,1.042)$ & 0.020 & $1.059(1.023,1.096)$ & $<0.001$ \\
\hline \multicolumn{5}{|l|}{ Stage } \\
\hline Stage III vs. I/II & $2.190(1.041,4.610)$ & 0.039 & Variable eliminated & \\
\hline Stage IV vs. I/II & $12.976(6.214,27.099)$ & $<0.001$ & Variable eliminated & \\
\hline $\mathrm{T}$ (3 and 4 vs. 1 and 2$)$ & Variable eliminated & & $3.365(1.956,5.788)$ & $<0.001$ \\
\hline $\mathrm{N}$ (positive vs. negative) & Variable eliminated & & $1.483(1.093,2.012)$ & 0.011 \\
\hline M (positive vs. negative) & Variable eliminated & & $2.520(0.851,7.460)$ & 0.095 \\
\hline Overall Cox model & & $<0.001$ & & $<0.001$ \\
\hline
\end{tabular}

Variable eliminated, variables that were not statistically significant were eliminated in the model. Overall Cox model, the model including all variables that were not eliminated. CI, confidence interval; EGR1, early growth response 1; HR, hazard rate.

addressing the TF-target regulatory spectrums of GC subtypes are required. In the present study, based on a publicly available GC cohort (25), genome-wide, condition-specific TF-target regulatory relationships of DF and IT GC were predicted using PANDA, by integrating known PPI, gene expression and sequence motif data of TFs. Furthermore, the biological function of NFYA in DF GC-derived MGC803 cells and IT GC-derived SGC-7901 cells was verified.

The results demonstrated that $>85 \%$ of TF-target regulatory relationships were shared among all DF, IT and MX subtypes of GC, suggesting strong conservation. It is therefore reasonable to suggest that TF-target edges were conserved, considering these networks were constructed using the same motif data. These results were also in accordance with previous studies using PANDA software $(22,24)$. Upon further investigation of the target genes of DF-specific and IT-specific edges, there were extremely small and limited overlaps with differentially expressed genes, which were identified in our previous study (16). This finding suggested that transcriptional alterations were predominantly caused by differential TF expression, rather than these specific TF-target regulatory relationships.

The majority of TFs enriched in DF-specific edges were also enriched in IT-specific edges, based on a hypergeometric distribution model. Therefore, to further reveal the differences in TF activity, the AnaPANDA algorithm was applied. A total of 13 TFs, including NFYA and FOXL1, were activated in DF GC, and eight TFs, including RELA and TLX1, were 
A

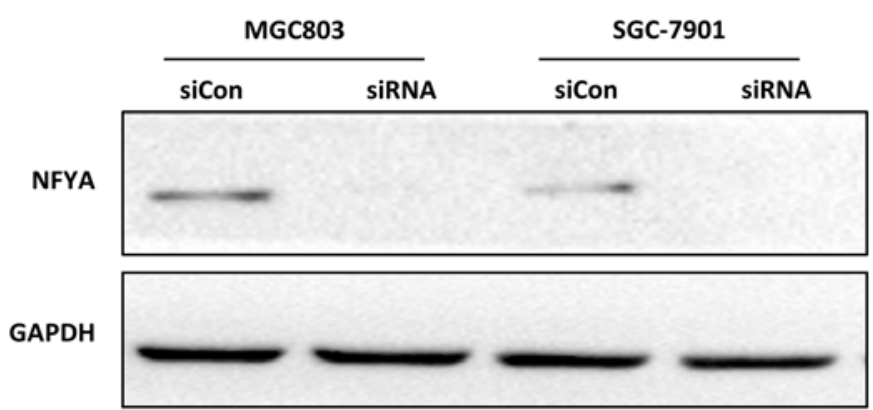

B
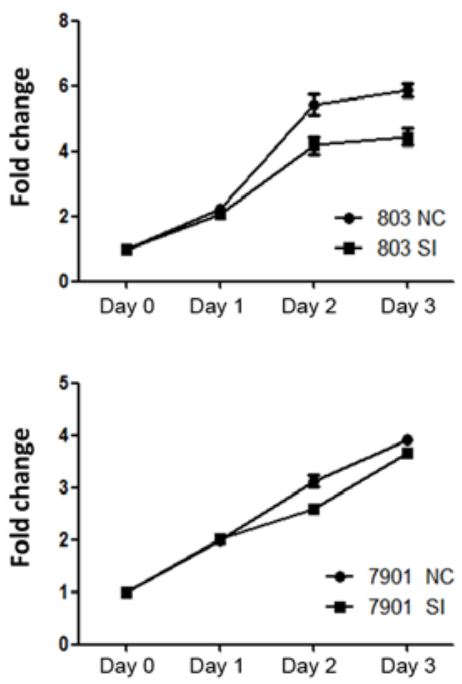

C
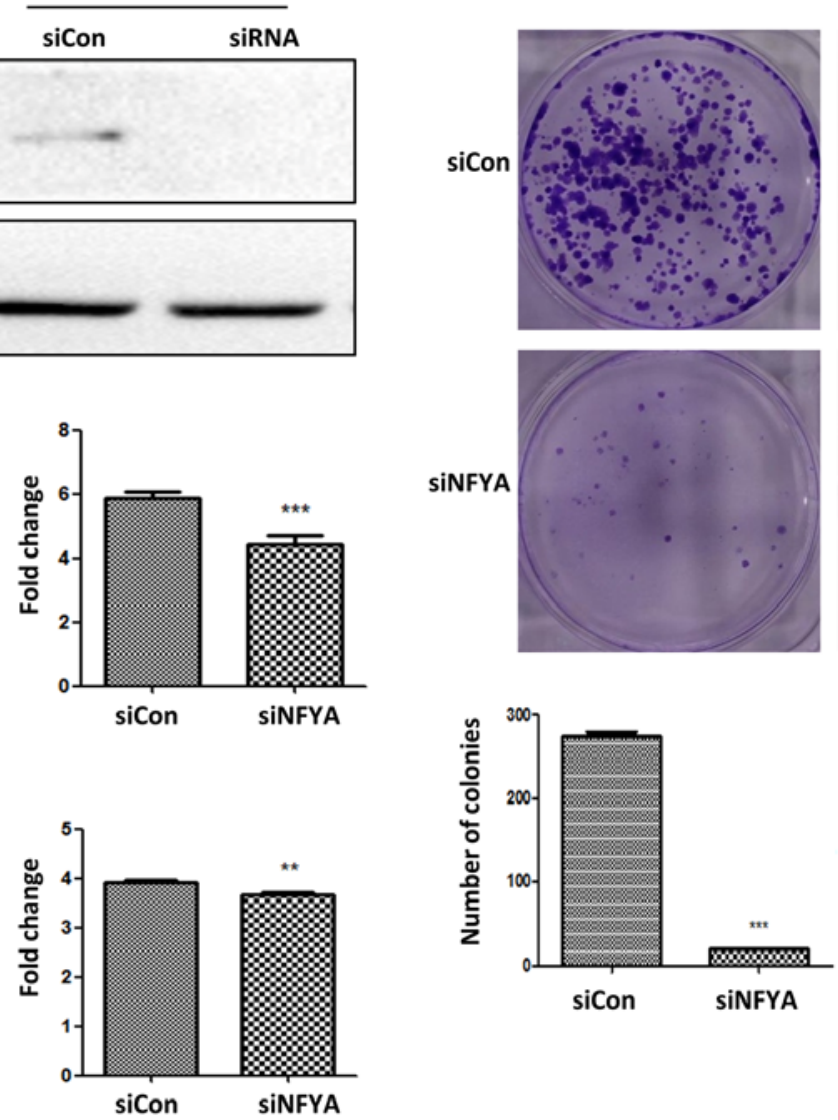

SGC-7901
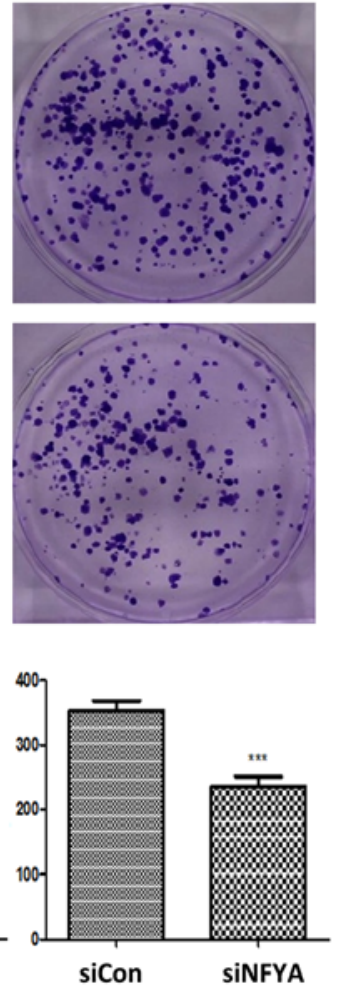

Figure 7. Investigation of the role of NFYA in diffuse and intestinal-derived GC cells. (A) Western blot analysis of NFYA knockdown by siRNA in MGC803 and SGC-7901 cells. (B) CCK-8 assay of MGC803 (upper panel) and SGC-7901 (lower panel) cell growth in the presence of siNFYA; the growth curve is presented on the left, and the fold change at day 3 is presented on the right. (C) Colony formation assays of MGC803 (left panel) and SGC-7901 (right panel) in the presence of siNFYA; representative colony formation results are presented in the upper panel, and the statistical results are presented in the lower panel. All assays were performed in triplicate. ${ }^{* *} \mathrm{P}<0.01 ;{ }^{* * *} \mathrm{P}<0.001$. NFYA, nuclear transcription factor Y subunit $\alpha$; siCon, control siRNA; siNFYA, NFYA siRNA; siRNA, small interfering RNA.

activated in IT GC. By evaluating these genes with survival analyses, four genes were identified as DF subtype-specific biomarkers and two genes were identified as IT subtypespecific biomarkers.

In the present study, NFYA, SRY, HAND1 and CREB1 were verified as DF-specific prognostic markers in GC. NFYA is one subunit of a TF complex, which has been demonstrated to activate metabolic pathways in cancer cells (37). SRY is a TF that initiates the development of male sex; SRY may also participate in cancer cell differentiation and the acquisition of cancer stem cell-like properties (38). HAND1 has an essential role in cardiac morphogenesis and has been confirmed as a biomarker in medulloblastoma (39), although, to the best of our knowledge, it has not been studied in other types of cancer. CREB1 may negatively regulate carbonic anhydrase IX in GC (40). In the present study, TLX1 and EGR1 were identified as IT-specific prognostic markers in GC. TLX1 participates in normal development of the spleen during embryogenesis. Dadi et al (41) reported that TLX1 is involved in tumor immunology processes, including T-cell maturation arrest in T-cell acute lymphoblastic leukaemia. EGR1 is a differentiation and mitogenesis-associated TF. In GC, EGR1 has been demonstrated to be important in tumor invasion, metastasis and heparanase transcription (42). Most of these TFs have not previously been considered as prognostic markers in GC. Therefore, the present findings provided novel insights into the discovery of specific biomarkers in certain subtypes of GC. Specifically, NFYA was selected for validation of biological function in DF GC-derived MGC803 cells and IT GC-derived SGC-7901 cells. Both CCK-8 and colony formation assays confirmed that knockdown of NFYA resulted in more marked effects on cell growth and colony formation in DF GC-derived cells, compared with IT GC-derived cells. This in vitro experiment further confirmed that NFYA was a specific independent prognostic factor in DF GC, but not in IT GC.

In conclusion, by combining network topologies and gene expression data, TF-target regulatory networks for DF, IT and MX GCs were constructed. It was demonstrated that different subtypes of GC contained different gene regulatory networks and TF activation patterns. Additionally, it was revealed that the same TFs had different biological effects in distinct GC subtypes. Specifically, NFYA was suggested as a DF subtypespecific independent prognostic factor in GC.

\section{Acknowledgements}

The authors would like to thank the Gene Expression Omnibus data repository for public access to the database. 


\section{Funding}

The present study was supported by the National Natural Science Foundation of China (grant nos. 81702314, 81670474).

\section{Availability of data and materials}

The datasets generated and analyzed during the current study are available in the Gene Expression Omnibus website (www. ncbi.nlm.nih.gov/geo/). Data regarding the biological function of NFYA in DF and IT GC analyzed during the current study are available from the corresponding author on reasonable request.

\section{Authors' contributions}

$\mathrm{BC}$ and LM performed the bioinformatics analysis. YZ carried out the cell line experiments. LM, SZhu and SZhang conceived and designed the study. YZ, HL, SG and JX helped to collect and reformat the primary data. XQ and $\mathrm{ZZ}$ helped to analyze the data and revised the manuscript. BC and LM drafted the manuscript. All authors read and approved the final manuscript.

\section{Ethics approval and consent to participate}

Not applicable.

\section{Patient consent for publication}

Not applicable.

\section{Competing interests}

The authors declare that they have no competing interests.

\section{References}

1. Torre LA, Bray F, Siegel RL, Ferlay J, Lortet-Tieulent J and Jemal A: Global cancer statistics, 2012. CA Cancer J Clin 65 : 87-108, 2015

2. Chen W, Zheng R, Baade PD, Zhang S, Zeng H, Bray F, Jemal A Yu XQ and He J: Cancer statistics in China, 2015. CA Cancer J Clin 66: 115-132, 2016.

3. Wong SS, Kim KM, Ting JC, Yu K, Fu J, Liu S, Cristescu R, Nebozhyn M, Gong L, Yue YG, et al: Genomic landscape and genetic heterogeneity in gastric adenocarcinoma revealed by whole-genome sequencing. Nat Commun 5: 5477, 2014.

4. Lauren T: The two histological main types of gastric carcinoma an attempt at a histoclinical classification. Acta Pathol Microbiol Scand 64: 19, 1965.

5. Hartgrink HH, Jansen EP, van Grieken NC and van de Velde CJ: Gastric cancer. Lancet 374: 477-490, 2009.

6. He Z and Li B: Recent progress in genetic and epigenetic profile of diffuse gastric cancer. Cancer Transl Med 1: 80-93, 2015.

7. Shah MA, Khanin R, Tang L, Janjigian YY, Klimstra DS, Gerdes H and Kelsen DP: Molecular classification of gastric cancer: A new paradigm. Clin Cancer Res 17: 2693-2701, 2011.

8. Fléjou JF: WHO Classification of digestive tumors: The fourth edition. Ann Pathol 31 (Suppl 5): S27-S31, 2011 (In French).

9. Palli D, Bianchi S, Cipriani F, Duca P, Amorosi A, Avellini C, Russo A, Saragoni A, Todde P and Valdes E: Reproducibility of histologic classification of gastric cancer. Br J Cancer 63 765-768, 1991.

10. Jinawath N, Furukawa $\mathrm{Y}$, Hasegawa S, Li M, Tsunoda T, Satoh S, Yamaguchi T, Imamura H, Inoue M, Shiozaki H, et al Comparison of gene-expression profiles between diffuse- and intestinal-type gastric cancers using a genome-wide cDNA microarray. Oncogene 23: 6830-6844, 2004.
11. Lee YS, Cho YS, Lee GK, Lee S, Kim YW, Jho S, Kim HM, Hong SH, Hwang JA, Kim SY, et al: Genomic profile analysis of diffuse-type gastric cancers. Genome Biol 15: R55, 2014.

12. Tanabe S, Aoyagi K, Yokozaki H and Sasaki H: Gene expression signatures for identifying diffuse-type gastric cancer associated with epithelial-mesenchymal transition. Int J Oncol 44: 1955-1970, 2014.

13. Förster S, Gretschel S, Jöns T, Yashiro M and Kemmner W: THBS4, a novel stromal molecule of diffuse-type gastric adenocarcinomas, identified by transcriptome-wide expression profiling. Mod Pathol 24: 1390-1403, 2011.

14. Kim B, Bang S, Lee S, Kim S, Jung Y, Lee C, Choi K, Lee SG, Lee K, Lee Y, et al: Expression profiling and subtype-specific expression of stomach cancer. Cancer Res 63: 8248-8255, 2003.

15. Yoon C, Cho SJ, Aksoy BA, Park DJ, Schultz N, Ryeom S and Yoon SS: Chemotherapy resistance in diffuse type gastric adenocarcinoma is mediated by RhoA activation in cancer stem-like cells. Clin Cancer Res 22: 971-983, 2016.

16. Min L, Zhao Y, Zhu S, Qiu X, Cheng R, Xing J, Shao L, Guo S and Zhang S: Integrated analysis identifies molecular signatures and specific prognostic factors for different gastric cancer subtypes. Transl Oncol 10: 99-107, 2017.

17. Ritchie MD, Holzinger ER, Li R, Pendergrass SA and Kim D: Methods of integrating data to uncover genotype-phenotype interactions. Nat Rev Genet 16: 85-97, 2015.

18. Vaquerizas JM, Kummerfeld SK, Teichmann SA and Luscombe NM: A census of human transcription factors: Function, expression and evolution. Nat Rev Genet 10: 252-263, 2009.

19. Buckingham M and Rigby PW: Gene regulatory networks and transcriptional mechanisms that control myogenesis. Dev Cell 28: 225-238, 2014.

20. Banks CA, Lee ZT, Boanca G, Lakshminarasimhan M, Groppe BD, Wen Z, Hattem GL, Seidel CW, Florens L and Washburn MP: Controlling for gene expression changes in transcription factor protein networks. Mol Cell Proteomics 13: 1510-1522, 2014.

21. Glass K, Huttenhower C, Quackenbush J and Yuan GC: Passing messages between biological networks to refine predicted interactions. PLoS One 8: e64832, 2013.

22. Lao T, Glass K, Qiu W, Polverino F, Gupta K, Morrow J, Mancini JD, Vuong L, Perrella MA, Hersh CP, et al: Haploinsufficiency of Hedgehog interacting protein causes increased emphysema induced by cigarette smoke through network rewiring. Genome Med 7: 12, 2015

23. Glass K, Quackenbush J, Spentzos D, Haibe-Kains B and Yuan GC: A network model for angiogenesis in ovarian cancer. BMC Bioinformatics 16: 115, 2015.

24. Min L, Zhang C, Qu L, Huang J, Jiang L, Liu J, Pinello L, Yuan GC and Shou C: Gene regulatory pattern analysis reveals essential role of core transcriptional factors' activation in triplenegative breast cancer. Oncotarget 8: 21938-21953, 2017.

25. Cristescu R, Lee J, Nebozhyn M, Kim K-M, Ting JC, Wong SS, Liu J, Yue YG, Wang J, Yu K, et al: Molecular analysis of gastric cancer identifies subtypes associated with distinct clinical outcomes. Nat Med 21: 449-456, 2015.

26. Irizarry RA, Hobbs B, Collin F, Beazer-Barclay YD, Antonellis KJ, Scherf U and Speed TP: Exploration, normalization, and summaries of high density oligonucleotide array probe level data. Biostatistics 4: 249-264, 2003.

27. Workman C, Jensen LJ, Jarmer H, Berka R and Gautier L: A new non-linear normalization method for reducing variability in DNA microarray experiments. Genome Biol: Aug 30, 2002 (Epub ahead of print)

28. Li C and Wong WH: Model-based analysis of oligonucleotide arrays: Expression index computation and outlier detection. Proc Natl Acad Sci USA 98: 31-36, 2001.

29. Wu C, Macleod I and Su AI: BioGPS and MyGene.info: Organizing online, gene-centric information. Nucleic Acids Res 41: D561-D565, 2013

30. Mathelier A, Fornes O, Arenillas DJ, Chen CY, Denay G, Lee J, Shi W, Shyr C, Tan G, Worsley-Hunt R, et al: JASPAR 2016: A major expansion and update of the open-access database of transcription factor binding profiles. Nucleic Acids Res 44: D110-D115, 2016.

31. Zhao Y, Min L, Xu C, Shao L, Guo S, Cheng R, Xing J, Zhu S and Zhang S: Construction of disease-specific transcriptional regulatory networks identifies co-activation of four gene in esophageal squamous cell carcinoma. Oncol Rep 38: 411-417, 2017. 
32. Ravasi T, Suzuki H, Cannistraci CV, Katayama S, Bajic VB, Tan K, Akalin A, Schmeier S, Kanamori-Katayama M, Bertin N, et al: An atlas of combinatorial transcriptional regulation in mouse and man. Cell 140: 744-752, 2010.

33. Ernst J, Beg QK, Kay KA, Balázsi G, Oltvai ZN and Bar-Joseph Z: A semi-supervised method for predicting transcription factorgene interactions in Escherichia coli. PLoS Comput Biol 4: e1000044, 2008

34. Lemmens K, Dhollander T, De Bie T, Monsieurs P, Engelen K, Smets B, Winderickx J, De Moor B and Marchal K: Inferring transcriptional modules from ChIP-chip, motif and microarray data. Genome Biol 7: R37, 2006.

35. Faith JJ, Hayete B, Thaden JT, Mogno I, Wierzbowski J, Cottarel G, Kasif S, Collins JJ and Gardner TS: Large-scale mapping and validation of Escherichia coli transcriptional regulation from a compendium of expression profiles. PLoS Biol 5: e8, 2007.

36. Altay G and Emmert-Streib F: Structural influence of gene networks on their inference: Analysis of C3NET. Biol Direct 6: 31, 2011.

37. Benatti P, Chiaramonte ML, Lorenzo M, Hartley JA, Hochhauser D, Gnesutta N, Mantovani R, Imbriano C and Dolfini D: NF-Y activates genes of metabolic pathways altered in cancer cells. Oncotarget 7: 1633-1650, 2016.

38. Murakami S, Ninomiya W, Sakamoto E, Shibata T, Akiyama H and Tashiro F: SRY and OCT4 are required for the acquisition of cancer stem cell-like properties and are potential differentiation therapy targets. Stem Cells 33: 2652-2663, 2015.
39. Asuthkar S, Gogineni VR, Rao JS and Velpula KK: Nuclear translocation of Hand-1 acts as a molecular switch to regulate vascular radiosensitivity in medulloblastoma tumors: The protein uPAR is a cytoplasmic sequestration factor for Hand-1. Mol Cancer Ther 13: 1309-1322, 2014.

40. Wang G, Cheng Z, Liu F, Zhang H, Li J and Li F: CREB is a key negative regulator of carbonic anhydrase IX (CA9) in gastric cancer. Cell Signal 27: 1369-1379, 2015.

41. Dadi S, Le Noir S, Payet-Bornet D, Lhermitte L, ZacariasCabeza J, Bergeron J, Villarèse P, Vachez E, Dik WA, Millien C, et al: TLX homeodomain oncogenes mediate T cell maturation arrest in T-ALL via interaction with ETS1 and suppression of TCR $\alpha$ gene expression. Cancer Cell 21: 563-576, 2012.

42. Zheng L, Pu J, Jiang G, Weng M, He J, Mei H, Hou X and Tong Q: Abnormal expression of early growth response 1 in gastric cancer: Association with tumor invasion, metastasis and heparanase transcription. Pathol Int 60: 268-277, 2010.

This work is licensed under a Creative Commons Attribution-NonCommercial-NoDerivatives 4.0 International (CC BY-NC-ND 4.0) License. 